\title{
QUANTITATIVE RESULTS FOR PERTURBED STOCHASTIC DIFFERENTIAL EQUATIONS
}

\author{
JORDAN STOYANOV and DOBRIN BOTEV \\ Bulgarian Academy of Sciences \\ Institute of Mathematics \\ 1090 Sofia, Box 373, Bulgaria
}

(Received August, 1995; Revised December, 1995)

\begin{abstract}
The paper is devoted to Itô type stochastic differential equations (SDE's) with "small" perturbations. Our goal is to present strong results showing how "close" are the $2 m$-order moments of the solutions of the perturbed SDE's and the unperturbed SDE.
\end{abstract}

Key words: Stochastic Differential Equation, "Small" Perturbations, Moments of Order 2m, Approximations of the Solutions.

AMS (MOS) subject classifications: $60 \mathrm{H} 10$.

\section{Introduction. Statement of the Problem}

The object of this study are stochastic differential equations (SDE's) of the following type

$$
X_{t}=X_{t_{0}}+\int_{t_{0}}^{t} a\left(s, X_{s}\right) d s+\int_{t_{0}}^{t} b\left(s, X_{s}\right) d w_{s}, \quad t \geq t_{0} \geq 0 .
$$

Here $w=\left(w_{t}, t \geq 0\right)$ is a standard Wiener process defined on a given probability space $(\Omega, \mathcal{F}, P)$, $a(t, x)$ and $b(t, \bar{x}), t \geq t_{0}, x \in \mathbb{R}^{1}$, are measurable real-valued functions, and $X_{t_{0}}$ is a random variable (r.v.) independent of $w$ with $E\left\{X_{t_{0}}^{2}\right\}<\infty$. Finally, $\int_{t_{0}}^{t} b(\cdot) d w_{s}$ is the well-known stochastic integral in Itô sense.

Under general conditions, the SDE (1) has a unique (strong) solution $X=\left(X_{t}, t \geq t_{0}\right)$, which is a diffusion Markov process with a drift coefficient $a$ and a diffusion coefficient $b^{2}$. Let us adopt the following classical conditions: For some constants $K_{1}>0$ and $K_{2}>0$ and all $t \geq t_{0}, x, y \in \mathbb{R}^{1}$ we have

$$
\left\{\begin{array}{c}
|a(t, x)-a(t, y)|+|b(t, x)-b(t, y)| \leq K_{1}|x-y| \\
a^{2}(t, x)+b^{2}(t, x) \leq K_{2}^{2}\left(1+x^{2}\right) .
\end{array}\right.
$$

Notice that standard references in the area of SDE's are the books by Gihman and Skorohod [4], Arnold [1], Liptser and Shiryaev [5] and Gard [3]. We shall systematically use basic facts from these sources without mentioning.

Now, along with (1), we consider another SDE: 


$$
X_{t}^{\epsilon}=X_{t_{0}}^{\epsilon_{0}}+\int_{t_{0}}^{t} \tilde{a}\left(s, X_{s}^{\epsilon}, \epsilon_{1}\right) d s+\int_{0}^{t} \tilde{b}\left(s, X_{s}^{\epsilon}, \epsilon_{2}\right) d w_{s}, \quad t \geq t_{0} .
$$

Here $\epsilon_{0}, \epsilon_{1}, \epsilon_{2}$ are "small" positive parameters, e.g., each is in the interval $(0,1]$ and $\epsilon$ stands for $\left(\epsilon_{0}, \epsilon_{1}, \epsilon_{2}\right) ; X_{t_{0}}^{\epsilon_{0}}, \tilde{a}$, and $\tilde{b}$ are as $X_{t_{0}}, a$, and $b$ above; $w$ is the same Wiener process. Thus the SDE (3) has also a unique (strong) solution $X^{\epsilon}=\left(X_{t}^{\epsilon}, t \geq t_{0}\right)$.

Our goal is to compare the solutions $X^{\epsilon}$ and $X$ of (3) and (1) in the case when their coefficients are related as follows:

$$
\left\{\begin{array}{c}
\tilde{a}\left(t, x, \epsilon_{1}\right)=a(t, x)+\alpha\left(t, x, \epsilon_{1}\right) \\
\tilde{b}\left(t, x, \epsilon_{2}\right)=b(t, x)+\beta\left(t, x, \epsilon_{2}\right)
\end{array}\right.
$$

The terms $\alpha(\cdot)$ and $\beta(\cdot)$ are called perturbations of the coefficients $a(\cdot)$ and $b(\cdot)$ which explains why (3) is called a "perturbed SDE" while the name "unperturbed SDE" is kept for (1).

Let us suppose that for some fixed natural number $m$ we have $E\left\{\left(X_{t_{0}}^{\epsilon_{0}}\right)^{2 m}\right\}<\infty$, $E\left\{\left(X_{t_{0}}\right)^{2 m}\right\}<\infty$ and let for all $t \geq t_{0}$

$$
\left\{\begin{array}{c}
E\left\{\left|X_{t_{0}}^{\epsilon_{0}}-X_{t_{0}}\right|^{2 m}\right\} \leq \delta_{0}\left(\epsilon_{0}\right) \\
\sup _{x}\left|\alpha\left(t, x, \epsilon_{1}\right)\right| \leq \delta_{1}\left(t, \epsilon_{1}\right) \\
\sup _{x}\left|\beta\left(t, x, \epsilon_{2}\right)\right| \leq \delta_{2}\left(t, \epsilon_{2}\right)
\end{array}\right.
$$

Thus we can expect that if the quantities $\delta_{0}\left(\epsilon_{0}\right), \delta_{1}\left(t, \epsilon_{1}\right), \delta_{2}\left(t, \epsilon_{2}\right)$ are small for small $\epsilon_{0}, \epsilon_{1}, \epsilon_{2}$, then the process $X^{\epsilon}$ is close to $X$. Recall that $X^{\epsilon}$ and $X$ are $2 m$-integrable in the sense that for each $t \geq t_{0}$ the r.v.'s $\left|X_{t}^{\epsilon}\right|^{2 m}$ and $\left|X_{t}\right|^{2 m}$ are $P$-integrable. Thus, the following quantity

$$
\Delta_{t}^{\epsilon}=E\left\{\left|X_{t}^{\epsilon}-X_{t}\right|^{2 m}\right\}
$$

is well-defined and we are interested in conditions guaranteeing that $\Delta_{t}^{\epsilon} \rightarrow 0$ as $\epsilon \rightarrow 0$. This means that for a fixed $t$, the $2 m$-order moments of $X_{t}^{\epsilon}$ and $X_{t}$ are close. Furthermore, we describe a few cases when $\Delta_{t}^{\epsilon} \rightarrow 0$ as $\epsilon \rightarrow 0$ on intervals whose length tend to infinity.

\section{Preliminary Result}

Let us prove first a result which is of independent interest. This result plays a key role for the statements in the next section.

Theorem A: Suppose conditions (2) and (5) are satisfied for the SDE's (1) and (3). Then for any $t \geq t_{0}$,

$$
\begin{gathered}
\Delta_{t}^{\epsilon} \leq\left\{\delta_{0}^{1 / m}\left(\epsilon_{0}\right) \exp \left[M\left(t-t_{0}\right)+2 \int_{t_{0}}^{t} \delta_{1}\left(s, \epsilon_{1}\right) d s\right]\right. \\
\left.+\int_{t_{0}}^{t}\left[2 \delta_{1}\left(s, \epsilon_{1}\right)+(2 m-1) \delta_{2}^{2}\left(s, \epsilon_{2}\right)\right] \exp \left[M(t-s)+2 \int_{s}^{t} \delta_{1}\left(\tau, \epsilon_{1}\right) d \tau\right] d s\right\}^{m},
\end{gathered}
$$


where $M=2 K+2(2 m-1) K^{2}\left(K=\max \left[K_{1}, K_{2}\right]\right.$, and $K_{1}$ and $K_{2}$ are the constants from (2)).

Proof: If we write explicitly the difference $Z_{t}^{\epsilon}=X_{t}^{\epsilon}-X_{t}, t \geq t_{0}$, apply the Itô formula to $\left(Z_{t}^{\epsilon}\right)^{2 m}$ and take expectations, we find that

$$
\Delta_{t}^{\epsilon}=E\left\{\left(Z_{t}^{\epsilon}\right)^{2 m}\right\}=E\left\{\left(Z_{t_{0}}^{\epsilon_{0}}\right)^{2 m}\right\}+2 m E\left\{I_{1}(t)\right\}+m(2 m-1) E\left\{I_{2}(t)\right\}+2 m E\left\{I_{3}(t)\right\}
$$

where

$$
\begin{aligned}
& I_{1}(t)=\int_{t_{0}}^{t}\left[\tilde{a}\left(s, X_{s}^{\epsilon}, \epsilon_{1}\right)-a\left(s, X_{s}\right)\right]\left(Z_{s}^{\epsilon}\right)^{2 m-1} d s \\
& I_{2}(t)=\int_{t_{0}}^{t}\left[\tilde{b}\left(s, X_{s}^{\epsilon}, \epsilon_{2}\right)-b\left(s, X_{s}\right)\right]^{2}\left(Z_{s}^{\epsilon}\right)^{2 m-2} d s \\
& I_{3}(t)=\int_{t_{0}}^{t}\left[\tilde{b}\left(s, X_{s}^{\epsilon}, \epsilon_{2}\right)-b\left(s, X_{s}\right)\right]\left(Z_{s}^{\epsilon}\right)^{2 m-1} d w_{s} .
\end{aligned}
$$

The existence of the $2 m$-order moments of $X_{t}^{\epsilon}$ and $X_{t}, t \geq t_{0}$ and the conditions on $\tilde{b}(\cdot)$ and $b(\cdot)$ allow us to use one of the properties of the stochastic integrals, thus concluding that

$$
E\left\{I_{3}(t)\right\}=0
$$

Let us estimate $I_{1}(t)$ and $I_{2}(t)$. In view of $(2)$, we see that

$$
I_{1}(t) \leq K \int_{t_{0}}^{t}\left|X_{s}^{\epsilon}-X_{s}\right|\left|Z_{s}^{\epsilon}\right|^{2 m-1} d s+\int_{t_{0}}^{t} \delta_{1}\left(s, \epsilon_{1}\right)\left|Z_{s}^{\epsilon}\right|^{2 m-1} d s .
$$

Now we take the expectations of both sides of the last inequality and applying Hölder's inequality to $E\left\{\left|Z_{s}^{\epsilon}\right|^{2 m-1}\right\}$ (see Shiryaev [7]) we find that

$$
E\left\{I_{1}(t)\right\} \leq \int_{t_{0}}^{t} \Delta_{s}^{\epsilon} d s+\int_{t_{0}}^{t} \delta_{1}\left(s, \epsilon_{1}\right)\left(\Delta_{s}^{\epsilon}\right)^{(2 m-1) /(2 m)} d s
$$

Similar arguments imply that

Therefore, from (7), (8) and (9), we get ${ }^{0}$

$$
E\left\{I_{2}(t)\right\} \leq 2 K^{2} \int_{t_{0}}^{t} \Delta_{s}^{\epsilon} d s+\int_{t_{0}}^{t} \delta_{2}^{2}\left(s, \epsilon_{2}\right)\left(\Delta_{s}^{\epsilon}\right)^{(m-1) / m} d s .
$$

$$
\begin{aligned}
\Delta_{t}^{\epsilon} \leq \delta_{0}\left(\epsilon_{0}\right) & +m M \int_{t_{0}}^{t} \Delta_{s}^{\epsilon} d s+2 m \int_{t_{0}}^{t} \delta_{1}\left(s, \epsilon_{1}\right)\left(\Delta_{s}^{\epsilon}\right)^{(2 m-1) /(2 m)} d s \\
& +m(2 m-1) \int_{t_{0}}^{t} \delta_{2}^{2}\left(s, \epsilon_{2}\right)\left(\Delta_{s}^{\epsilon}\right)^{(m-1) / m} d s .
\end{aligned}
$$

Now we use the following elementary inequality 


$$
v^{r} \leq v^{r}+v
$$

which is valid for any nonnegative number $v$ and $0<r_{1} \leq r_{2}<1$. Setting

$$
r_{1}=(m-1) / m, \quad r_{2}=(2 m-1) /(2 m), \text { and } v=\Delta_{s}^{\epsilon}
$$

we find that

$$
\left(\Delta_{s}^{\epsilon}\right)^{(2 m-1) /(2 m)} \leq\left(\Delta_{s}^{\epsilon}\right)^{(m-1) / m}+\Delta_{s}^{\epsilon}
$$

and hence (10) takes the form

$$
\begin{gathered}
\Delta_{t}^{\epsilon} \leq \delta_{0}\left(\epsilon_{0}\right)+m \int_{t_{0}}^{t}\left[M+2 \delta_{1}\left(s, \epsilon_{1}\right)\right] \Delta_{s}^{\epsilon} d s \\
+\int_{t_{0}}^{t}\left[2 m \delta_{1}\left(s, \epsilon_{1}\right)+m(2 m-1) \delta_{2}^{2}\left(s, \epsilon_{2}\right)\right]\left(\Delta_{s}^{\epsilon}\right)^{(m-1) / m} d s .
\end{gathered}
$$

The last tool we need is the following generalized Gronwall-Bellman inequality (see Filatov and Sharova [2]):

If a nonnegative function $u(t), t \geq t_{0}$, satisfies the integral inequality

$$
u(t) \leq C+\int_{t_{0}}^{t} A(s) u(s) d s+\int_{t_{0}}^{t} B(s)[u(s)]^{\gamma} d s
$$

where $C \geq 0,0 \leq \gamma<1$, and functions $A(t)$ and $B(t), t \geq t_{0}$, are nonnegative and continuous, then

$$
u(t) \leq\left\{C^{1-\gamma} \exp \left[(1-\gamma) \int_{t_{0}}^{t} A(s) d s\right]+(1-\gamma) \int_{t_{0}}^{t} B(s) \exp \left[(1-\gamma) \int_{s}^{t} A(\tau) d \tau\right] d s\right\}^{1 /(1-\gamma)} .
$$

Obviously, it remains to apply this inequality to (11) by letting

$$
\begin{gathered}
u(t)=\Delta_{t}^{\epsilon}, \quad C=\delta_{0}\left(\epsilon_{0}\right), \quad \gamma=(m-1) / m, \\
A(s)=m\left[M+2 \delta_{1}\left(s, \epsilon_{1}\right)\right], \\
B(s)=2 m \delta_{1}\left(s, \epsilon_{1}\right)+m(2 m-1) \delta_{2}^{2}\left(s, \epsilon_{2}\right) .
\end{gathered}
$$

Thus we arrive at the desired relation (6). Theorem A is proved.

\section{Basic Results. Proofs}

Since the magnitude of the perturbations of SDE (1) is determined by the quantities $\delta_{0}\left(\epsilon_{0}\right)$, $\delta_{1}\left(t, \epsilon_{1}\right)$ and $\delta_{2}\left(t, \epsilon_{2}\right)$ (see (4) and (5)) it is natural to impose some conditions on these quantities and see how $\Delta_{t}^{\epsilon}=E\left\{\left|X_{t}^{\epsilon}-X_{t}\right|^{2 m}\right\} \rightarrow 0$ as $\epsilon \rightarrow 0$ and on which intervals this convergence holds. 
Three specific cases will be considered.

In the statements below (Theorems 1,2 and 3) we assume (with mentioning it again) that the general conditions of Theorem A are satisfied. We also use the constant $M=2 K+$ $2(2 m-1) K^{2}$.

Theorem 1: Suppose that for all $t \geq t_{0}$,

$$
\delta_{0}\left(\epsilon_{0}\right)=\epsilon_{0}, \delta_{1}\left(t, \epsilon_{1}\right)=\epsilon_{1} \text { and } \delta_{2}\left(t, \epsilon_{2}\right)=\epsilon_{2}
$$

Define the numbers $\epsilon$ and $T_{1}$ as follows:

$$
\epsilon=\max \left[\epsilon_{0}^{1 / m}, \epsilon_{1}, \epsilon_{2}^{2}\right] \text { and } T_{1}=(1-\rho) /(M+\rho),
$$

where $\rho \in(0,1)$ is arbitrarily chosen.

Then the following relation holds:

$$
\sup _{t} \Delta_{t}^{\epsilon \rightarrow 0} \text { as } \epsilon \rightarrow 0 \text { for } t \in\left[t_{0}, t_{0}+T_{1} \ln (1 / \epsilon)\right) \text {. }
$$

Proof: In Theorem A we have found the upper bound (6) for $\Delta_{t}^{\epsilon}$ and now by using (12) we can go further. After a substitution we arrive at

$$
\left(\Delta_{t}^{\epsilon}\right)^{1 / m} \leq \epsilon_{0}^{1 / m} \exp \left[M\left(t-t_{0}\right)+2 \epsilon_{1}\left(t-t_{0}\right)\right]+\int_{t_{0}}^{t}\left[2 \epsilon_{1}+(2 m-1) \epsilon_{2}^{2} \exp \left[M(t-s)+2 \epsilon_{1}(t-s)\right]\right] d s .
$$

Since $\epsilon \rightarrow 0$ implies that $\epsilon_{1} \rightarrow 0$, we can assume that $2 \epsilon_{1}<\rho$. Taking into account that $\epsilon_{0}^{1 / m} \leq \epsilon, \epsilon_{1} \leq \epsilon$ and $\epsilon_{2}^{2} \leq \epsilon$ we find that

$$
\left(\Delta_{t}^{\epsilon}\right)^{1 / m} \leq C_{1} \epsilon e^{(M+\rho) t}+(4 \epsilon) /(M+\rho)
$$

where the constant $C_{1}$ depends on $t_{0}$ and $m$ but not on $t$.

Obviously, (13) implies that $\Delta_{t}^{\epsilon} \rightarrow 0$ as $\epsilon \rightarrow 0$ for each $t$ on any finite interval $\left[t_{0}, t_{1}\right]$ with fixed $t_{1}>t_{0}$. However, we can use (13) and make one step further by extending the time-interval on which $\Delta_{t}^{\epsilon \rightarrow 0}$. Indeed, if we take $T_{1}=(1-\rho) /(M+\rho)$ we find from (13) that

$$
\Delta_{t}^{\epsilon} \leq\left[C_{1} \epsilon^{\rho}+(4 \epsilon) /(M+\rho)\right]^{m} \text { for any } t \in\left[t_{0}, t_{0}+T_{1} \ln (1 / \epsilon)\right)
$$

and hence $\sup _{t} \Delta_{t}^{\epsilon} \rightarrow 0$ as $\epsilon \rightarrow 0$ on the interval $\left[t_{0}, t_{0}+T_{1} \ln (1 / \epsilon)\right)$. Note that the length of this interval tends to infinity as $\epsilon \rightarrow 0$. Theorem 1 is proved.

Theorem 2: Suppose that $t_{0}>1$ and let for all $t \geq t_{0}$,

$$
\delta_{0}\left(\epsilon_{0}\right)=t_{0}^{-1 / \epsilon_{0}}, \delta_{1}\left(t, \epsilon_{1}\right)=t^{-1 / \epsilon_{1}} \text { and } \delta_{2}\left(t, \epsilon_{2}\right)=t^{-1 / \epsilon_{2}} .
$$

Define $\epsilon$ and $T_{2}$ as follows:

$$
\epsilon=\max \left[m \epsilon_{0}, \epsilon_{1}, \epsilon_{2} / 2\right] \text { and } T_{2}=(1 / M) \ln \left(t_{0}-\rho\right),
$$

where $\rho$ is an arbitrary number in the interval $\left(0, t_{0}\right)$.

Then,

$$
\sup _{t} \Delta_{t}^{\epsilon} \rightarrow 0 \text { as } \epsilon \rightarrow 0 \text { for } t \in\left[t_{0}, t_{0}+T_{2} / \epsilon\right) \text {. }
$$


Proof: From (6) and (14), we find that

$$
\begin{gathered}
\left(\Delta_{t}^{\epsilon}\right)^{1 / m} \leq t_{0}^{-1 /\left(m \epsilon_{0}\right)} \exp \left[M\left(t-t_{0}\right)+2 \epsilon_{1}\left(t_{0}^{1-1 / \epsilon_{1}}-t^{1-1 / \epsilon_{1}}\right) /\left(1-\epsilon_{1} 0\right]\right. \\
+\int_{t_{0}}^{t}\left[2 s^{-1 / \epsilon_{1}}+(2 m-1) s^{-2 / \epsilon_{2}}\right] \exp \left[M(t-s)+2 \epsilon_{1}\left(s^{1-1 / \epsilon_{1}}-t^{1-1 / \epsilon}\right) /\left(1-\epsilon_{1} 0\right] d s .\right.
\end{gathered}
$$

Obviously, we can take $\epsilon_{1}<1 / 2$, and, since $s^{-1 /\left(m \epsilon_{0}\right)} \leq s^{-1 / \epsilon}, s^{-1 / \epsilon_{1}} \leq s^{-1 / \epsilon}$ and $s^{-2 / \epsilon_{2}} \leq s^{-1 / \epsilon}$ for any $s>1$, we derive that

Hence,

$$
\left(\Delta_{t}^{\epsilon}\right)^{1 / m} \leq t_{0}^{-1 / \epsilon} e^{M\left(t-t_{0}\right)+2}+4 m \int_{t_{0}}^{t} s^{-1 / \epsilon} e^{M(t-s)+2} d s .
$$

$$
\left(\Delta_{t}^{\epsilon}\right)^{1 / m} \leq C_{2} t_{0}^{-1 / \epsilon} e^{M t}
$$

where $C_{2}$ is a constant depending on $t_{0}$ and $m$ but not on $t$. It follows from (15) that $\Delta_{t}^{\epsilon} \rightarrow 0$ as $\epsilon \rightarrow 0$ for each $t$ on any finite interval $\left[t_{0}, t_{2}\right]$ with fixed $t_{2}>t_{0}$. Moreover, with $T_{2}=$ $(1 / M) \ln \left(t_{0}-\rho\right)$ we easily find that

$$
\Delta_{t}^{\epsilon} \leq C_{2}^{m}\left(1-\rho / t_{0}\right)^{m / \epsilon} \text { for all } t \in\left[t_{0}, t_{0}+T_{2} / \epsilon\right) .
$$

Since $0<1-\rho / t_{0}<1$, the conclusion is that $\sup _{t} \Delta_{t}^{\epsilon} \rightarrow 0$ as $\epsilon \rightarrow 0$ on the interval $\left[t_{0}, t_{0}+T_{2} / \epsilon\right)$ whose length tends to infinity. Theorem 2 is proved.

Theorem 3: Suppose that $t_{0}>0$ and let for all $t \geq t_{0}$

$$
\delta_{0}\left(\epsilon_{0}\right)=e^{-t_{0} / \epsilon_{0}}, \delta_{1}\left(t, \epsilon_{1}\right)=e^{-t / \epsilon_{1}} \text { and } \delta_{2}\left(t, \epsilon_{2}\right)=e^{-t / \epsilon_{2}} .
$$

For an arbitrary $\rho \in\left(0, t_{0}\right)$, define

$$
\epsilon=\max \left[m \epsilon_{0}, \epsilon_{2} / 2\right] \text { and } T_{3}=\left(t_{0}-\rho\right) / M .
$$

Then,

$$
\sup _{t}^{\epsilon} \Delta_{t}^{\epsilon} \rightarrow 0 \text { as } \epsilon \rightarrow 0 \text { for } t \in\left[t_{0}, t_{0}+T_{3} / \epsilon\right)
$$

Proof: Taking $\epsilon_{1}<1$ (we can do this since $\epsilon_{1} \rightarrow 0$ ) we easily see that $\int_{t_{0}}^{t} e^{-s / \epsilon_{1}} d s<1$ and $\int_{s}^{t} e^{-\tau / \epsilon_{1}} d \tau<1$. Then, substituting (16) into (6) and suitably transforming the right-hand side of (6) we finally arrive at the relation:

$$
\left(\Delta_{t}^{\epsilon}\right)^{1 / m} \leq C_{3} e^{M t-t_{0} / \epsilon}
$$

where $C_{3}$ depends on $t_{0}, m$ and $M$ but not on $t$.

Therefore, $\Delta_{t}^{\epsilon} \rightarrow 0$ as $\epsilon \rightarrow 0$ for each $t$ on any finite interval $\left[t_{0}, t_{3}\right]$ with fixed $t_{3}>t_{0}$. Even more, if $T_{3}=\left(t_{0}=\rho\right) / M$, then

$$
\sup _{t} \Delta_{t}^{\epsilon} \leq C_{3}^{m} e^{-\rho / \epsilon} \rightarrow 0 \text { as } \epsilon \rightarrow 0 \text { for } t \in\left[t_{0}, t_{0}+T_{3} / \epsilon\right) .
$$


Again the convergence to zero holds on intervals whose lengths tends to infinity. Theorem 3 is proved.

\section{Additional Remarks}

(a) In Theorems 1, 2 and 3 not only did we prove that $\sup _{t} \Delta_{t}^{\epsilon} \rightarrow 0$ as $\epsilon \rightarrow 0$ but, in addition, we can specify the rate of convergence. It is a power rate in Theorem 1 and exponential rate in Theorems 2 and 3. Moreover, we can specify the rate of getting to infinity of the lengths of the corresponding intervals. Obviously, both rates depend on the magnitude of perturbations.

(b) Instead of $\Delta_{t}^{\epsilon}=E\left\{\left|X_{t}^{\epsilon}-X_{t}\right|^{2 m}\right\}$, we can consider the quantity

$$
\tilde{\Delta}_{t}^{\epsilon}=E\left\{\sup _{t \in\left[t_{0}, T\right]}\left|X_{t}^{\epsilon}-X_{t}\right|^{2 m}\right\}
$$

as a measure of closeness between the processes $X^{\epsilon}$ and $X$. If we establish a sup-version of Theorem $\mathrm{A}$ and use some additional arguments, we can provide conditions under which $\widetilde{\Delta}_{T}^{\epsilon} \rightarrow 0$ as $\epsilon \rightarrow 0$ on finite fixed intervals or on intervals whose lengths tend to infinity.

(c) The results of the present paper can be used when studying stability properties of SDE's under perturbations. Another possibility is to look for the so-called expansions of the solution $X^{\epsilon}$ of the perturbed SDE (3) assuming some smoothness of the coefficients $\tilde{a}(\cdot)$ and $\tilde{b}(\cdot)$.

(d) Similar questions can be raised for more general SDE's driven by arbitrary semimartingales not just by the standard Wiener process (see Protter [6]).

\section{Acknowledgement}

One of the authors (J.S.) is grateful to Professor Tom Stroud (Queen's University, Kingston, Canada) for his attention and support. We are grateful to the anonymous referee and the associate editor for their useful comments.

\section{References}

[1] Arnold, L. Stochastic Differential Equations. Theory and Applications, John Wiley, New York 1974.

[2] Filatov, A. and Sharova, L., Integral Inequalities and Theory of the Nonlinear Vibrations, Nauka, Moscow 1976 (in Russian).

[3] Gard, T., Introduction to Stochastic Differential Equations, Marcel Dekker, New York 1988.

[4] Gihman, I and Skorohod, A., Stochastic Differential Equations, Springer-Verlag, Berlin 1972.

[5] Lipster, R. and Shiryaev, A., Statistic of Random Processes, Vol. 1, Springer-Verlag, New York 1977.

[6] Protter, Ph., Stochastic Integration and Differential Equations: A New Approach, Springer-Verlag, New York 1990.

[7] Shiryaev, A., Probability, 2nd edition, Springer-Verlag, New York 1995. 


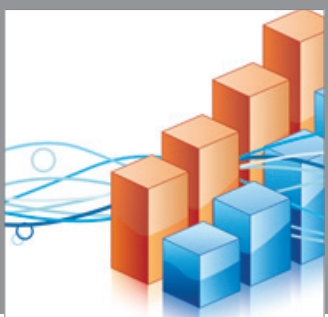

Advances in

Operations Research

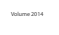

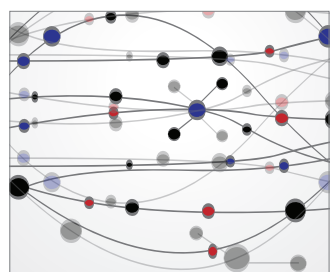

\section{The Scientific} World Journal
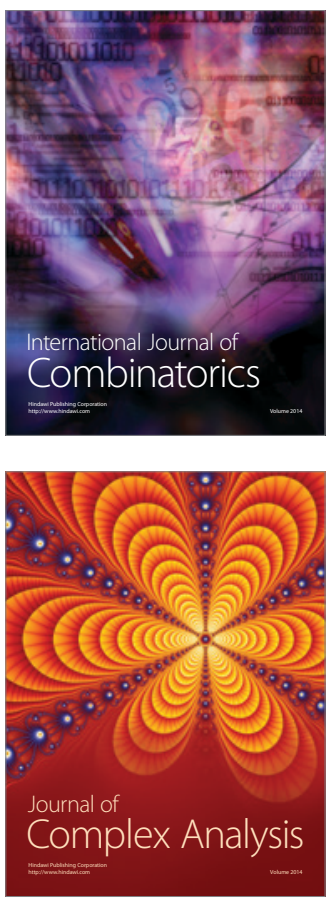

International Journal of

Mathematics and

Mathematical

Sciences
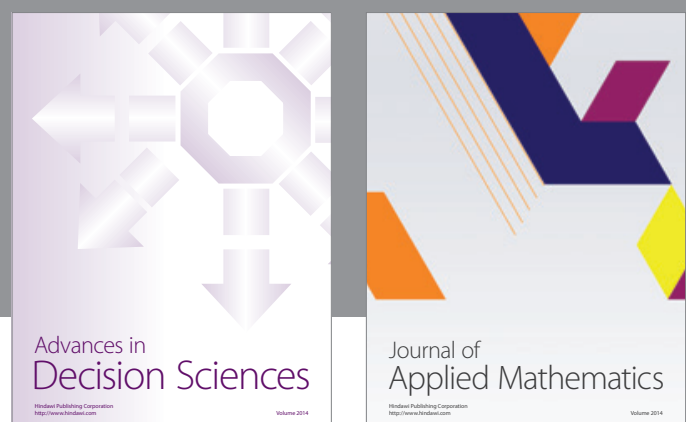

Journal of

Applied Mathematics
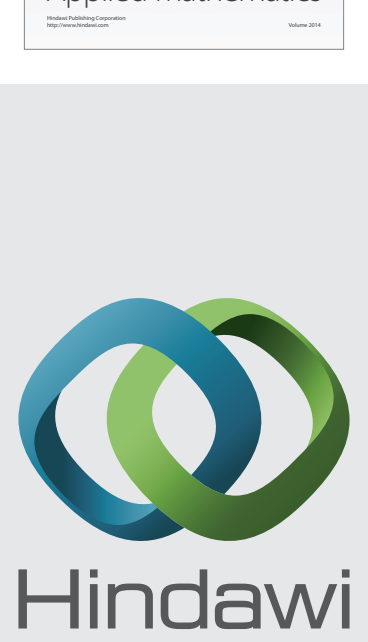

Submit your manuscripts at http://www.hindawi.com
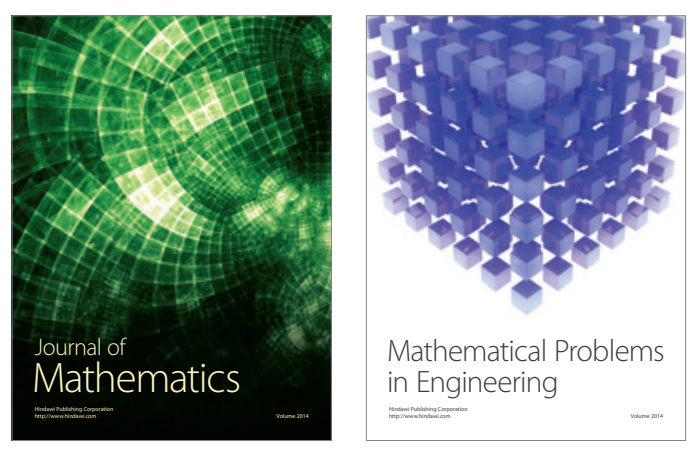

Mathematical Problems in Engineering
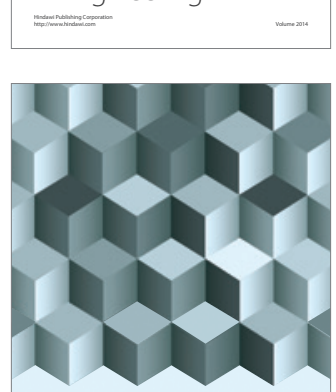

Journal of

Function Spaces
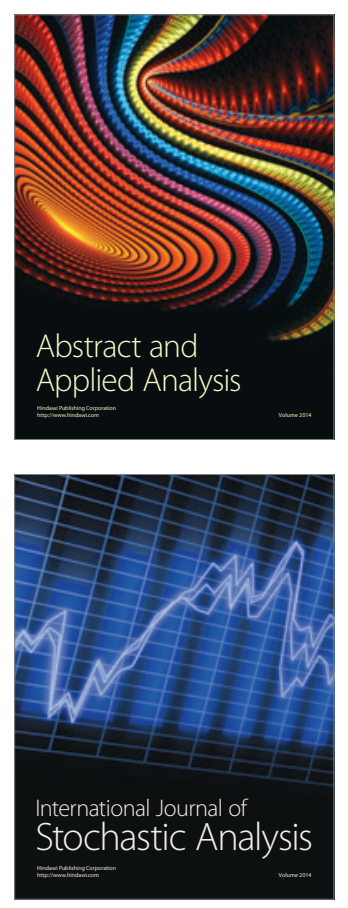

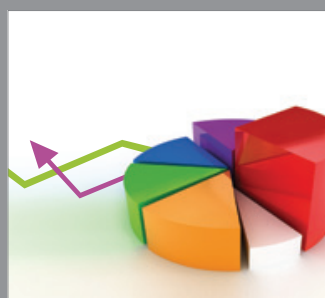

ournal of

Probability and Statistics

Promensencen
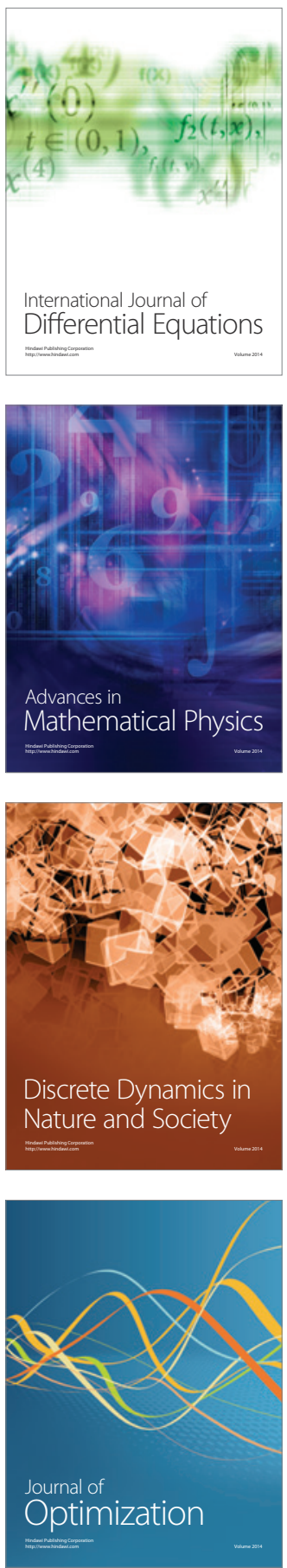